\title{
The safety and clinical efficacy of recombinant human granulocyte colony stimulating factor injection for colon cancer patients undergoing chemotherapy
}

\author{
JIE Chen ${ }^{1}$, YIN PAN ${ }^{2 *}$
}

'Northern Jiangsu People's Hospital, Jiangsu, China

${ }^{2}$ Clinic Medical College of Jilin University, Jilin, China

Study conducted at Clinic Medical College of Jilin University, Jilin, China

Article received: $4 / 25 / 2017$ Accepted for publication: 5/7/2017

*Correspondence: Jilin - China Postal code: 130000 hqmmary@163.com

http://dx.doi.org/10.1590/1806-9282.63.12.1061

\section{SUMMARY}

Objective: The present study was designed to evaluate safety and efficacy of recombinant human granulocyte colony stimulating factor (G-CSF) injection and whether this regimen could reduce the incidence of adverse events caused by chemotherapy.

Method: A total of 100 patients with colon cancer who were treated with chemotherapy in our hospital from January 2011 to December 2014 were randomly divided into two groups, with 50 patients in each group. The patients in the treatment group received G-CSF 24 hours after chemotherapy for consecutive three days; the patients in the control group received the same dose of normal saline. Routine blood tests were performed 7 days and 14 days after chemotherapy.

Results: Compared with the control group, the incidences of febrile neutropenia and leukocytopenia in the treatment group were significantly lower $(\mathrm{p}<0.05)$. In addition, the incidence of liver dysfunction in the treatment group was lower than that of the control group, without statistical significance. The incidence of myalgia in the treatment was higher than that of the control group without statistical significance.

Conclusion: The present study indicated that G-CSF injection after chemotherapy is safe and effective for preventing adverse events in colon cancer patients with chemotherapy.

Keywords: granulocyte-macrophage colony-stimulating factor, drug therapy, febrile neutropenia.

\section{INTRODUCTION}

Although advanced screening and preventative strategies have been widely applied in recent years, colorectal carcinoma remains a major public health problem worldwide. ${ }^{1}$ Currently, surgical resection of the primary colorectal lesions combined with adjuvant chemotherapy and radiation is the mainstay of treatment. ${ }^{2}$ However, despite recent advances in earlier detection and improvements in chemotherapy, the median survival for all patients with metastatic colorectal carcinoma is approximately $22-24$ months, with a 5 -year survival rate $<5 \%{ }^{3}$

Leukopenia is one of the most common adverse events and dosage-limiting toxicities of chemotherapy for patients with colon cancer. In addition, it results in febrile neutropenia (FN) which is related to life-threatening infections with a mortality rate of $7-11 \%{ }^{4-6}$ The previous study has shown that granulocyte colony stimulating factor (G-CSF) could decrease the incidence of leukopenia and FN, and, therefore, infections and infection-related mortality, as well as the incidence of chemotherapy dosage reductions and regimen delays. ${ }^{7}$ Usually, G-CSF is recommended after chemotherapy until complete recovery of leukocyte levels. Weycker et al. ${ }^{8}$ have shown that prophylactic application of G-CSF resulted in a one-third to two-third decrease in the risk of re-hospitalization due to FN in patients treated with chemotherapy. In addition, two more recent clinical 
studies $^{8,9}$ on comparative effectiveness of G-CSF prophylaxis yielded similar findings. In China, G-CSF has been widely used to reduce chemotherapy-induced leukopenia. However, the clinical efficacy and safety of prophylactic application of G-CSF for Chinese colon cancer patients have not been fully studied yet. Therefore, we designed and conducted the present comparative study. A total of 100 patients with colon cancer who were treated with chemotherapy were randomly divided into two groups, with 50 patients in each group. The treatment group received G-CSF prophylaxis 24 hours after chemotherapy for three consecutive days. The control group received G-CSF with the same amount of normal saline.

\section{MethOD}

\section{Patient eligibility}

All of the patients had their diagnosis of metastatic colorectal adenocarcinomas confirmed by pathology and/ or cytology. They all underwent chemotherapy in Subei People's Hospital (Yangzhou, Jiangsu, China) from January 2011 to December 2014. Inclusion criteria were: (1) Karnofsky performance status $\geq 60$; (2) age between 18-75 years; (3) predicted survival time $\geq 3$ months; (4) adequate bone marrow functions indicated by white blood cell counts $>4.0 \times 10^{9}$ and transaminases $<1.5$ times and within the upper limit of normal; (5) without heart, liver and kidney dysfunctions. Exclusion criteria were: (1) unability to complete two cycles of chemotherapy; (2) any severe medical and/or psychiatric conditions; (3) presence of any other malignancies; (4) women who were pregnant or breastfeeding at the time of study.

The present study was reviewed and approved by the Medical Ethics Committee of West China Hospital (Cheng$\mathrm{du}$, Sichuan, China). All participants provided written consent forms.

\section{Treatment method}

A total of 100 eligible patients were randomly divided into two groups. Patients in the treatment group received subcutaneous administration of G-CSF (JiSaiXin, Huabei Jintan pharmaceutical Co.) 24 hours after chemotherapy, $150 \mu \mathrm{g}$ per day, for three consecutive days. Patients in the control group received the same amount of normal saline. Routine blood tests were performed 7 days and 14 days after chemotherapy.

Adverse events were evaluated using the National Cancer Institute Common Terminology Criteria for Adverse Events, v3.0. Dose-limiting toxicity (DLT) was defined as grade $3 / 4$ non-hematologic adverse events and grade 4 hematologic adverse events.

\section{Statistical analysis}

IBM SPSS 17.0 statistical software was used to analyze the data. Continuous data were expressed as mean \pm standard deviation (SD). Categorical data were expressed as percentages. Intergroup comparisons were performed using Chi-square test (or Fisher's exact test) or variance analysis or rank sum test. All the tests were performed using a two-sided test of difference, where the inspection level $\alpha$ of 0.05 and a difference with $p<0.05$ were considered statistically significant.

\section{RESULTS}

\section{Patient characteristics}

As shown in Table 1, a total of 100 patients were enrolled in the present study including 50 in the treatment group and 50 in the control group. All patients received at least two cycles of chemotherapy prior to the enrollment. In the treatment group, the age range was between 25 and 77 years with a mean age of 57 years. There were 30 male and 20 female patients. In the control group, the age range was between $27-75$ years, with a mean age of 57 years. There were 29 male and 21 female patients.

TABLE 1 General clinical characteristics of patients.

\begin{tabular}{lll} 
& Treatment group & Control group \\
\hline Median age (range, years) & $57(25-77)$ & $58(27-75)$ \\
\hline Gender & & \\
\hline Male & 30 & 29 \\
\hline Female & 20 & 21 \\
\hline Treatment & \\
\hline No prior chemotherapy & 37 & 33 \\
\hline With prior chemotherapy & 13 & 17 \\
\hline Primary tumor site & & \\
\hline Liver cancer & 20 & 19 \\
\hline Lung cancer & 16 & 12 \\
\hline Other & 14 & 19
\end{tabular}

\section{Adverse events}

In the treatment group, there was no patient diagnosed with FN, in other words, an FN incidence of $0 \%$. In the control group, there were three patients diagnosed with $\mathrm{FN}$, yielding an $\mathrm{FN}$ incidence of $6 \%$. In addition, in the treatment group, there were 17 patients diagnosed with leukopenia grade I/II and one patient diagnosed with leukopenia grade III/IV with the leukopenia incidence of $36 \%$. In addition, there were 17 patients diagnosed with leukopenia grade I/II and one patient diagnosed with leukopenia grade III/IV with the leukopenia incidence of $36 \%$. In the control group, there were 27 patients diagnosed with leu- 
kopenia grade I/II and nine patients diagnosed with leukopenia grade III/IV with a leukopenia incidence of $72 \%$. The incidences of FN and leukopenia were significantly lower in the treatment group compared with that of the control group (both p-values $<0.05$ ). The differences in incidence of liver dysfunction, renal function abnormality, shock, acute interstitial pneumonia and myalgia were not statistically significant between the two groups (all p-values $>0.05$ ). Adverse events were shown in Table 2.

TABLE 2 Adverse events of Chinese colon cancer patients with prior chemotherapy with or without G-CSF administration.

\begin{tabular}{lll} 
Adverse events & Treatment group & Control group \\
\hline Febrile neutropenia & 0 & 3 \\
\hline Leukopenia & & \\
\hline Grade I/II & 17 & 27 \\
\hline Grade III/IV & 1 & 9 \\
\hline Myalgia & 3 & 2 \\
\hline Liver dysfunction & 7 & 8 \\
\hline Renal function abnormality & 0 & 0 \\
\hline Shock & 0 & 0 \\
\hline Acute interstitial pneumonia & 0 & 0 \\
\hline
\end{tabular}

\section{Toxicity}

No DLTs were observed. Local reactions at injection sites including erythema, induration, and pruritus were observed in all patients, and tenderness in five of 100 patients. Minimal systemic toxicities were observed in the treatment group (Table 3 ). The most common adverse effects were transient, low-grade fever/chill (14\%), fatigue (8\%), nausea $(6 \%)$ and headache (12\%). Fever and chills were likely due to G-CSF treatment, while nausea was probably the result of cyclophosphamide administration. Headache may have been the result of ondansetron, which was provided as an optional and prophylactic antiemetic before the administration of cyclophosphamide. Therefore, the dose level we used in the present study was considered to have an acceptable degree of safety and warrants subsequent trials to further assess G-CSF associated clinical outcomes.

\section{TABLE 3 G-CSF-related systemic adverse reactions.}

\begin{tabular}{llll}
$\begin{array}{l}\text { Adverse } \\
\text { reactions }\end{array}$ & $\begin{array}{l}\text { CTC } \\
\text { grade }\end{array}$ & $\begin{array}{l}\text { No. of } \\
\text { events }\end{array}$ & $\begin{array}{l}\text { Incidences of adverse } \\
\text { reactions (\%) }\end{array}$ \\
\hline Fever/chill & 1 & 7 & 14 \\
\hline Headache & 1 & 6 & 12 \\
\hline Fatigue & 1 & 4 & 8 \\
\hline Nausea & 1 & 3 & 6 \\
\hline CTC: common terminology criteria for adverse reactions.
\end{tabular}

\section{Discussion}

The present study has demonstrated the clinical efficacy and safety of G-CSF for Chinese patients with metastatic colorectal cancer. To our knowledge, this was the first study of a G-CSF for colorectal cancer patients.

As one of the main side-effects of chemotherapy, bone marrow suppression has serious impacts on clinical efficacy and safety for patients with malignancies undergoing chemotherapy. As a specific hematopoietic regulating growth factor of granulocyte lineage, G-CSF can be produced by gene recombination technique and therefore become recombinant human granulocyte colony stimulating factor. ${ }^{10-12}$ It promotes the multipotent hemopoietic stem cells to differentiate into mature granulocytes and macrophages in peripheral blood. ${ }^{13}$

Based on the American Society of Clinical Oncology (ASCO) recommendations for the use of white blood cell growth factors in $2006^{14}$ and the National Comprehensive Cancer Network (NCCN $)^{15}$ guidelines for the use of myeloid growth factors in 2013, G-CSF's primary prophylactic use, secondary prophylactic use and therapeutic use were stated as follows. Primary prophylactic use was recommended for patients based on the risks of developing FN. Secondary prophylactic use was recommended for patients who had developed neutropenic complications from a prior cycle of chemotherapy. Therapeutic use should be considered for patients with fever and neutropenia who were at high risk of infection-associated complications or who had prognostic factors which predicted poor clinical outcomes. In addition, patient's age, chemoradiotherapy regimen, pneumonia, hypotension, and nutritional status were not factors to determine whether or not influence use G-CSF.

The present study has demonstrated that the incidences of FN and leukopenia were significantly lower in the treatment group compared with that of the control group (both p-values $<0.05$ ), with the incidences of FN being $0 \%$ in the treatment group and $6 \%$ in the control group, and the incidences of leukopenia being $36 \%$ in the treatment group and $72 \%$ in the control group. The differences of incidences of liver dysfunction, renal function abnormality, shock, acute interstitial pneumonia and myalgia were not statistically significant between the two groups (all p-values > 0.05).

Although G-CSF alone as an adjuvant therapy showed some promise in colon cancer patients, ${ }^{16-18}$ its role as an adjuvant therapy when combined with chemotherapy remains to be determined. The present study described a novel adjuvant therapy for colorectal cancer chemotherapy. The present study found it to be safe and well toler- 
ated for Chinese patients with colon cancer undergoing chemotherapy to receive subcutaneously administration of G-CSF 24 hours after chemotherapy $150 \mu \mathrm{g}$ per day for consecutive three days.

However, there were some limitations in the present study. Firstly, the present study was conducted using relatively small sample size of patients in a single institution. Therefore, multiple-centered studies with larger sample size of penitents will be needed to verify our results and conclusions. Secondly, in the present study, G-CSF administration started 24 hours after chemotherapy and lasted for three consecutive days based on our clinical experience. However, the best regimen for G-CSF administration has been unknown. Therefore, we recommended further studies to verify our results and conclusion and determine the best regimen for G-CSF administration.

\section{Conclusion}

In conclusion, our study suggests that prophylactic administration of G-CSF 24 hours after chemotherapy $150 \mu \mathrm{g}$ per day for three consecutive days is a safe and effective treatment for preventing FN and leukopenia in Chinese patients with colon cancer undergoing chemotherapy.

\section{RefERENCES}

1. Jemal A, Center MM, DeSantis C, Ward EM. Global patterns of cancer incidence and mortality rates and trends. Cancer Epidemiol Biomarkers Prev. 2010; 19(8):1893-907.

2. Benson AB 3rd, Bekaii-Saab T, Chan E, Chen YJ, Choti MA, Cooper HS, et al.; National Comprehensive Cancer Network. Localized colon cancer, version 3.2013: featured updates to the NCCN Guidelines. J Natl Compr Canc Netw. 2013; 11(5):519-28.

3. Benson AB 3rd, Bekaii-Saab T, Chan E, Chen YJ, Choti MA, Cooper HS, et al. Metastatic colon cancer, version 3.2013: featured updates to the NCCN Guidelines. J Natl Compr Canc Netw. 2013; 11(2):141-52.

4. Caggiano V, Weiss RV, Rickert TS, Linde-Zwirble WT. Incidence, cost, and mortality of neutropenia hospitalization associated with chemotherapy. Cancer. 2005; 103(9):1916-24.
5. Kuderer NM, Dale DC, Crawford J, Lyman GH. Impact of primary prophylaxis with granulocyte colony-stimulating factor on febrile neutropenia and mortality in adult cancer patients receiving chemotherapy: a systematic review. J Clin Oncol. 2007; 25(21):3158-67.

6. Lal A, Bhurgri Y, Rizvi N, Virwani M, Memon RU, Saeed W, et al. Factors influencing in-hospital length of stay and mortality in cancer patients suffering from febrile neutropenia. Asian Pac J Cancer Prev. 2008; 9(2):303-8.

7. Liou SY, Stephens JM, Carpiuc KT, Feng W, Botteman MF, Hay JW. Economic burden of haematological adverse effects in cancer patients: a systematic review. Clin Drug Investig. 2007; 27(6):381-96.

8. Weycker D, Barron R, Edelsberg J, Kartashov A, Oster G. Comparative effectiveness of filgrastim, pegfilgrastim, and sargramostim as prophylaxis against hospitalization for neutropenic complications in patients with cancer receiving chemotherapy. Am J Clin Oncol. 2012; 35(3):267-74.

9. Tan H, Tomic K, Hurley D, Daniel G, Barron R, Malin J. Comparative effectiveness of colony-stimulating factors for febrile neutropenia: a retrospective study. Curr Med Res Opin. 2011; 27(1):79-86.

10. Thomas MC, Greten TF, Pardoll DM, Jaffee EM. Enhanced tumor protection by granulocyte-macrophage colony-stimulating factor expression at the site of an allogeneic vaccine. Hum Gene Ther. 1998; 9(6):835-43.

11. Dranoff G, Jaffee E, Lazenby A, Golumbek P, Levitsky H, Brose K, et al. Vaccination with irradiated tumor cells engineered to secrete murine granulocytemacrophage colony-stimulating factor stimulates potent, specific, and longlasting anti-tumor immunity. Proc Natl Acad Sci USA. 1993; 90(8):3539-43.

12. Borrello I, Sotomayor EM, Cooke S, Levitsky HI. A universal granulocytemacrophage colony-stimulating factor-producing bystander cell line for use in the formulation of autologous tumor cell-based vaccines. Hum Gene Ther. 1999; 10(12):1983-91.

13. Cui L, Liu XX, Jiang Y, Liu JJ, Zhou XR, He XJ, et al. Phase II study on dose escalating schedule of paclitaxel concurrent with radiotherapy in treating patients with locally advanced non-small cell lung cancer. Asian Pac J Cancer Prev. 2014; 15(4):1699-702.

14. Smith TC, Khatcheressian J, Lvman GH, Ozer H, Armitage JO, Balducci L, et al. 2006 update of recommendations for the use of white blood cell growth factors: an evidence-based clinical practice guideline. J Clin Oncol. 2006; 24(19):3187-205

15. Crawford J, Armitage J, Balducci L, Becker PS, Blayney DW, Cataland SR, et al.; National Comprehensive Cancer Network. Myeloid growth factors. J Natl Compr Canc Netw. 2013; 11(10):1266-90.

16. Ju H, Xing W, Yang J, Zheng Y, Jia X, Zhang B, et al. An effective cytokine adjuvant vaccine induces autologous $T$-cell response against colon cancer in an animal model. BMC Immunol. 2016;17(1):31.

17. Narusawa M, Inoue H, Sakamoto C, Matsumura Y, Takahashi A, Inoue T, et al. TLR7 ligand augments GM-CSF-initiated antitumor immunity through activation of plasmacytoid dendritic cells. Cancer Immunol Res. 2014; 2(6):568-80.

18. Correale P, Fioravanti A, Bertoldi I, Montagnani F, Miracco C, Francini G. Occurrence of autoimmunity in a long-term survivor with metastatic colon carcinoma treated with a new chemo-immunotherapy regimen. J Chemother. 2008; 20(2):278-81 